\title{
A Non-Contact Ultrasonic Platform for Structural Inspection
}

\author{
Gordon Dobie, Member, IEEE, Rahul Summan, Student Member, IEEE, S. Gareth Pierce, Walter Galbraith, \\ Gordon Hayward, Fellow, IEEE
}

\begin{abstract}
Miniature robotic vehicles are receiving increasing attention for use in non-destructive testing (NDE) due to their attractiveness in terms of cost, safety and their accessibility to areas where manual inspection is not practical. Conventional ultrasonic inspection requires the provision of a suitable coupling liquid between the probe and the structure under test. This necessitates either an on board reservoir or umbilical providing a constant flow of coupling fluid, neither of which are practical for a fleet of miniature robotic inspection vehicles. Air-coupled ultrasound offers the possibility of couplant-free ultrasonic inspection. This paper describes the sensing methodology, hardware platform and algorithms used to integrate an air-coupled ultrasonic inspection payload into a miniature robotic vehicle platform. The work takes account of the robot's inherent positional uncertainty when constructing an image of the test specimen from aggregated sensor measurements. The paper concludes with the results of an automatic inspection of a aluminium sample.
\end{abstract}

Index Terms-robotics, NDE, sensor platforms

\section{INTRODUCTION}

The industrialised world is full of structural assets that need to be periodically inspected to prevent unscheduled and costly outages or catastrophic failures. Such failures can lead to large scale environmental disasters and loss of life. Ideally such inspection should determine the structural integrity of a component without testing it to breaking point. This is termed Non-Destructive Evaluation (NDE) and is achieved by testing the component for degradation, using specific measurements that detect problems such as cracks, corrosion, or impact damage. Ideally NDE can be performed in-situ, often while the component is in use, but operational conditions may preclude this.

Inspections are generally performed manually, with trained operators scanning the structure with handheld probes. 'Human factors' are often the weakest component in NDE inspections [1] with overwork, lack of sufficient training and monotonous inspections leading to inconsistent quality [2]. Furthermore, human access to the inspection site is often difficult or intrinsically unsafe. Inspection sites are commonly located in inconvenient locations, partially obstructed, or in uncomfortable working environments. Access may require extensive scaffolding, or plant shutdowns. In the most extreme cases, like those found in the nuclear industry, radioactive or chemical contamination prohibits human access altogether.

Centre for Ultrasonic Engineering

Department of Electronic and Electrical Engineering,

University of Strathclyde,

204 George Street, Glasgow, G1 1XW, UK

E-mail: (see http://www.cue.ac.uk/staff.html).
Miniature Remote Sensing Agents (RSAs) are well suited for the inspection of many structures and installations. Onboard power and wireless communications obviates the need for umbilical cabling maximising access and mobility. Their small size and magnetic traction makes them particularly useful for inspecting areas with limited access. It allows them to crawl over the surface of a structure, along pipes, and into small spaces. Moreover, the concept can be extended to a fleet of heterogeneous robots which improves the functionality, reliability and flexibility of the system as a whole. Such a fleet consists of multiple RSAs, each carrying one of a range of NDE inspection measurement payloads based on ultrasonic, electromagnetic or visual techniques. Flexibility is achieved by adjusting the size and consistency of the fleet. For example, one robot may be used to inspect a pipe section, five robots may be used to inspect a small boiler and one hundred robots may be used to inspect the hull of a ship. Reliability is achieved through division of labour and redundancy; no individual robot being crucial to the inspection. The system can be optimised in real time, based on new inspection results or a change in operating conditions. The various inspection payloads can be used in a complementary manner. For example, visual inspection provides detailed information about the surface, whereas ultrasound can penetrate into the material, albeit with coarser resolution. Lastly, the individual robots can be made to be low-cost and even disposable, with complex capabilities emerging through the use of a fleet, rather than through individual complexity. This paper considers a specific type of miniature robot, non-contact ultrasonic inspection device. Unlike conventional ultrasound sensors which generate and measure ultrasonic bulk waves the embedded payload makes use of guided ultrasonic waves called Lamb Waves [3] which propagate in plate like structures. Guided waves offer significant potential for the following reasons:

1) Lamb waves can be generated with non-contact air coupled angled transducers [4].

2) When generating the zero-order antisymmetric Lamb wave mode in the dispersive region, the excitation angle and operating frequency of the angled transducer are dependent on the test specimen's material thickness. If the excitation frequency is constant, the thickness can be calculated by sweeping the incident angle. Alternatively the incident angle can be fixed and the frequency swept.

3) It is possible to rapidly scan large areas by propagating Lamb waves over long distances.

This paper begins by describing the miniature robot plat- 
form and its non-contact ultrasonic payload. Robot positional uncertainty is introduced and sample results of an extended Kalman filter used for guidance are presented. An algorithm is introduced that combines sensor measurements while taking into consideration the robots positional uncertainty. The paper concludes with the results of an automatic inspection.

\section{SySTEM DESIGN}

\section{A. Vehicle Architecture}

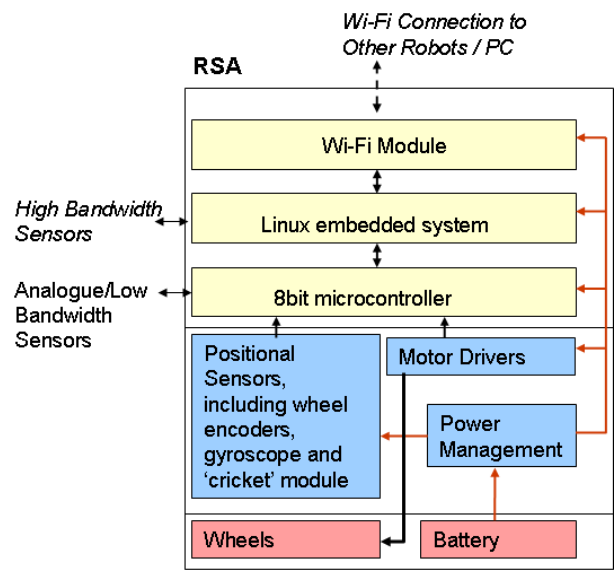

Fig. 1. System diagram of the miniature robot

Each RSA is a differentially driven vehicle with a passive castor wheel. The current prototype is approximately $14 \mathrm{~cm} \mathrm{x}$ $14 \mathrm{~cm} \times 10 \mathrm{~cm}$ and has magnetic wheels for traction on inclined ferromagnetic surfaces. A block diagram of the vehicle is shown in Figure 1 and a photograph is shown in Figure 2.

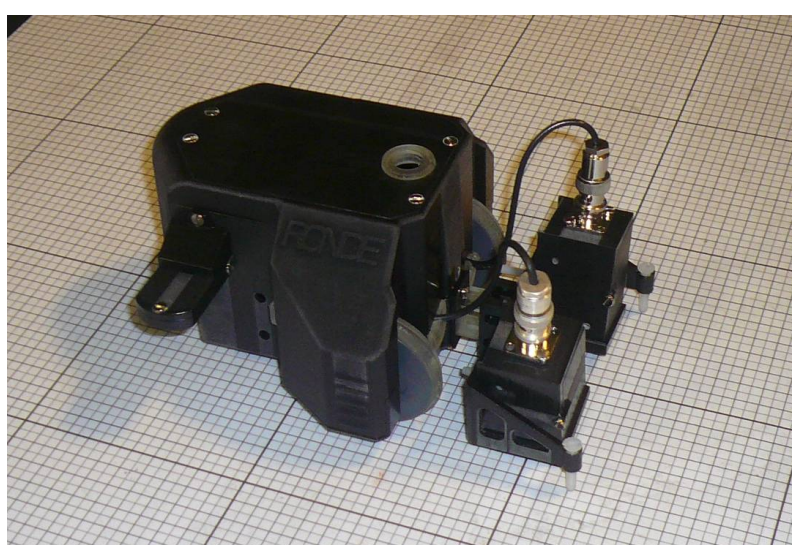

Fig. 2. Ultrasonic inspection robot showing air-coupled ultrasonic payload. The bold lines have $100 \mathrm{~mm}$ spacing.

An 8 bit microcontroller performs low level vehicle control. A gyroscope and two optical 300 count quadrature encoders are interfaced with the microcontroller and are used to calculate the robot's relative position and for closed-loop-control of the two motors. The 'Cricket' ultrasonic positioning system [5] provides absolute positional measurements. A Kalman filter [6] combines these absolute and relative measurements to give each RSA accurate positional information. A $400 \mathrm{MHz}$ Linux computer acts as the main RSA processor and performs high level movement calculations that are fed to the microcontroller as a series of simple manoeuvres. The Linux computer can perform on-board processing of results and interface with high bandwidth sensors such as a miniature camera or the ultrasonics payload. The robotic fleet is inter-connected using a high bandwidth ad-hoc WiFi network.

\section{B. The Air-Coupled Transducer Concept}

Ultrasonic inspection allows the interrogation of the inner volume of the structure under test allowing it to detect defective regions hidden from other sensing techniques. The technology is amenable to large area scanning, an important issue for cost effective monitoring of larger structures. However conventional ultrasonic inspection requires the provision of a suitable coupling liquid between the probe and the structure under test. Maintaining the supply and consistency of coupling is problematic for the type of mobile system under consideration and this is also compounded by the need to minimise the size of the sensor platforms.

An alternative approach is to use air as the coupling fluid and through optimisation of the transducer and associated electronics, compensate for the inevitable loss of sensitivity, which can be as much as $30 \mathrm{~dB}$ when compared with a conventional liquid coupled system due to the impedance mismatch between air and the transducer or sample. The operating frequency is usually restricted to below $1 \mathrm{MHz}$, due to the high attenuation in air and accordingly, resolution is reduced due to the longer wavelengths in the test material. However, previous work by some of the authors and others, [7], [8] has shown the validity of through air, Lamb wave ultrasonic inspection. Here a pair of air coupled ultrasonic transducers were inclined at an appropriate angle to the test specimen, to generate the zeroth order anti-symmetric $\left(A_{0}\right)$ Lamb wave in both plate and curved metallic structures. The $A_{0}$ Lamb wave mode is dispersive, with the phase velocity dependent on the material properties and the frequency thickness product [3]. A change in plate thickness, due to erosion or otherwise, results in attenuation of the Lamb wave signal at the receiving transducer and it is by this mechanism that defects are detected. Crack type defects may also scatter the Lamb wave further attenuating the received signal. It should be noted that Lamb wave propagation is a function of the specimen's elastic properties and that factors, such as structural loading, will effect Lamb wave propagation. However, these changes are typically negligible when compared to the effect of defects.

The Lamb wave can propagate forward for a considerable distance (in the order of metres) from the transmitter allowing the system to be used in several different configurations which are detailed in Figure 3. In the local pitch-catch configuration shown in Figure 3 'A' both transducers are mounted on a single robot and examine the area underneath the transducers. The inspection area is approximately equal to the combined surface area of the two transducers. This configuration typically measures signal attenuation. The second configuration shown in Figure 3 ' $\mathrm{B}$ ' is the extended pitch-catch arrangement. In this case, the transducers are split over two robots, one carrying the transmitter, the other the receiver. This extends the 

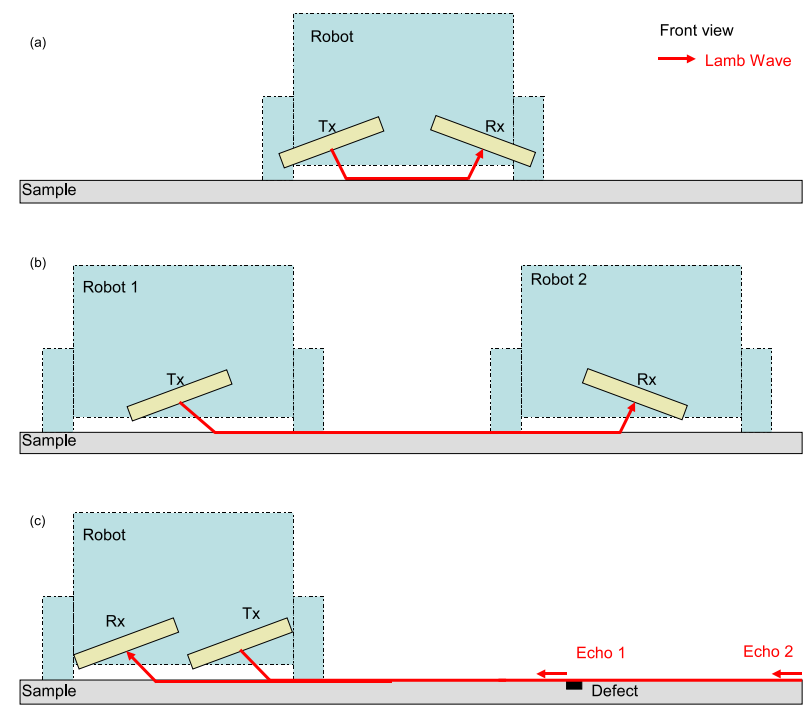

Fig. 3. Schematic of three possible air-coupled Lamb wave inspection configurations. 'A' Local pitch-catch using a single robot. 'B' Extended pitchcatch using two robots. 'C' Pulse-echo using a single robot.

coverage to a strip between the robots. In this method, either signal attenuation or time of flight can be measured. In the third configuration shown in Figure 3 ' $\mathrm{C}$ ' the transducers are configured for pulse-echo. The large dynamic range between transmit and receive signals makes it impractical to perform pulse-echo measurements with a single transducer (receiver signals are masked by transmit ringing), so pitch-catch transducers are used to synthesise a pulse-echo transducer. This configuration measures the time it takes for the pulse to travel to and from a reflector. This time is typically converted to a distance using the wave propagation velocity. The current work concentrates on the local pitch-catch configuration, the two remaining configurations will be considered in subsequent papers.

Alternative non-contact methods of ultrasonic inspection include laser techniques [9] and the use of Electromagnetic Acoustic Transducers (EMATs) [10]. Laser generated ultrasound can excite and record a large number of modes, but are typically too large and power demanding for integration into the robot platform. EMATs can excite and detect a large variety of Lamb wave modes [10] depending on the coil design. They are robust and can work at high temperature, but generally have low sensitivity and require the test material to be conductive. Additionally EMAT transmitters require a high drive current making it difficult to embed them into the RSAs. EMATs receivers show some potential for use on the vehicles as omnidirectional sensors, but have not been investigated in this work.

\section{Ultrasonic PAyloAd Design}

\section{A. The Physics of Angled Air-Coupled Ultrasound Transduc-} ers

The air-coupled transducer arrangement in shown in Figure 4. When the transmitter is excited, the axial mode produces a planar wave radiating from the front face. Efficient matching ensures most of the energy radiates from the front of the transducer [11]. Also, if a 1-3 piezoelectric composite is used, the composite nature of the transducer helps to dampen out unwanted radial modes. The angle $\theta_{i}$ is selected to phase match the transducer output to the desired Lamb wave mode as described by Snell's Law [12]. For propagation in a $1 \mathrm{~mm}$ thick aluminium plate at $600 \mathrm{kHz}$, the appropriate value of $\theta_{i}$ $=9.8$ degrees $\left(c_{p h} \simeq 2000 \mathrm{~m} / \mathrm{s}\right)$.

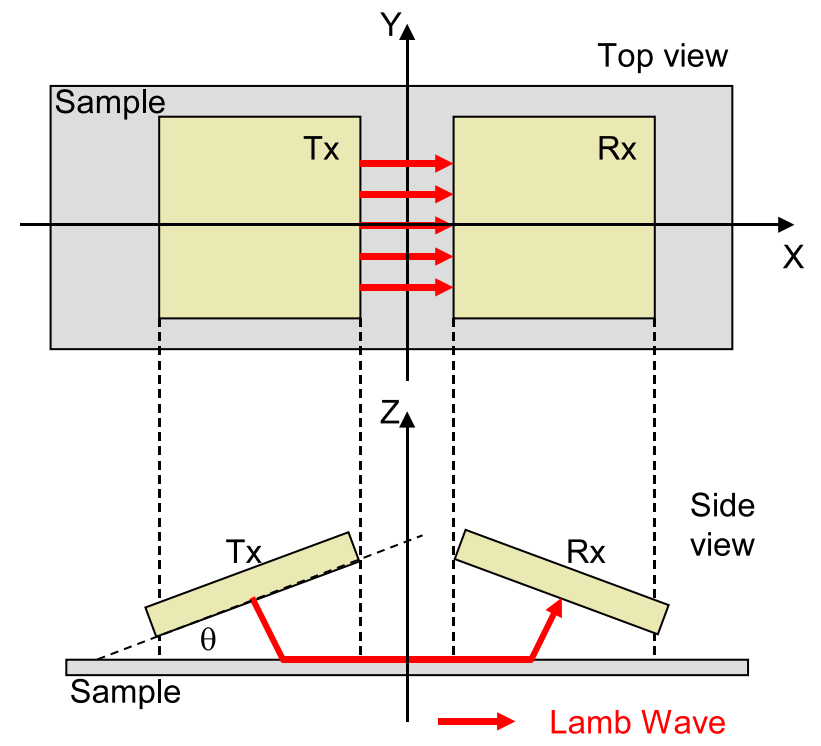

Fig. 4. Schematic diagram of local pitch-catch experiment showing general layout of the sensors.

\section{B. Hardware Design}

The embedded non-contact ultrasonic inspection payload is capable of exciting and measuring $600 \mathrm{kHz} A_{0}$ Lamb waves in thin steel or aluminium plates. The $A_{0}$ Lamb wave mode generates significantly more out-of-plane displacement than other modes which makes it suitable for generation from angled transducers. This operating frequency was chosen as a trade off between temporal/spatial resolution and signal attenuation (and therefore SNR) of the system. Piezoelectric composite transducers were selected for their improved sensitivity.

The payload electronics consist of three components; the payload controller (including ADC), the transducer front end and the receiver preamplifier. These are illustrated in Figure 5 and discussed in Sections III-D, III-E and III-F respectively. Section III-G evaluates the payload's performance.

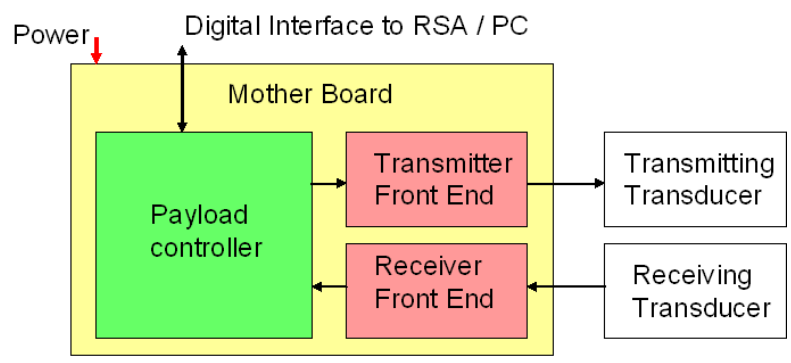

Fig. 5. Block diagram of ultrasonics payload 


\begin{tabular}{|c|l|l|l|l|} 
ine Layer & $\begin{array}{l}\text { Thickness } \\
(\mathrm{mm})\end{array}$ & $\begin{array}{l}\text { Density } \\
\left(\mathrm{kgm}^{-3}\right)\end{array}$ & $\begin{array}{l}\text { Atten } \\
1 \\
\left(\mathrm{MHCm}^{-1}\right)\end{array}$ & $\begin{array}{l}\text { Elastic } \\
\text { Stiffness } \\
\left(\mathrm{Nm}^{-3}\right)\end{array}$ \\
ine 1. SR & Variable & 951 & 10 & $9.41 \mathrm{e} 8$ \\
2. MSR & 0.08 & 951 & 18 & $3.09 \mathrm{e} 8$ \\
3. SF & 0.01 & 697 & 100 & $6.97 \mathrm{e} 8$ \\
4. MF & 0.11 & 360 & 120 & $4.2 \mathrm{e} 7$ \\
ine & & & &
\end{tabular}

TABLE I

CHARACTERISTICS OF THE MATCHING LAYER'S SUB-LAYERS.

\section{Payload Transducers}

The payload uses a pair of $30 \mathrm{~mm}$ square 1-3 piezoelectric composite transducers designed to operate at the desired $600 \mathrm{kHz}$, in pitch-catch mode. Following the transducer design guidelines of Kelly [13], the transmitting transducer had a 70\% volume fraction of PZT-5H and the receiving transducer had a $30 \%$ volume fraction of PZT-5A. In both cases, the passive filler material was CY1301/HY1300. A matching layer was integrated onto the front-face of each transducer to minimise insertion loss due to the impedance miss-match between the transducer face and air. The matching layer was created by curing a thin layer of silicon rubber onto membrane filter paper which gradually reduced the acoustic impedance as the energy passed from the silicon through to the porous paper and into the air.

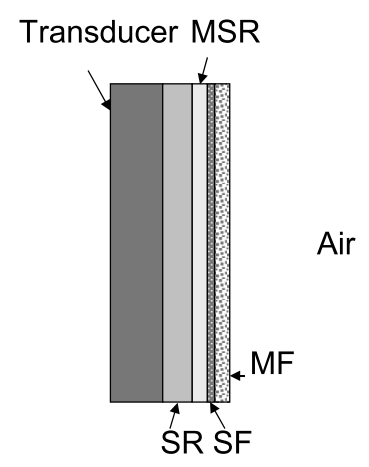

Fig. 6. Illustration of multilayer matching to air

Kelly et al [11] used an electron microscope to examine this design and modelled the matching layer as four sub layers, these are shown in Figure 6. The transducer interfaces with a layer of silicon rubber (SR). A portion of the silicon rubber is modified by the presence of the membrane filter (MF) and is termed modified silicon rubber (MSR). The third layer is a result of the membrane filter absorbing silicon rubber and is termed 'saturated filter' (SF). The thickness of the MSR, $\mathrm{SF}$ and MF were found to be constant and these thickness are documented in Table III-C. The thickness of the silicon rubber is optimised for minimal insertion loss and following the advice of Kelly et al, was set to $0.2 \mathrm{~mm}$ for transmission and $0.64 \mathrm{~mm}$ for reception. The material properties of each layer is shown in Table III-C.

By integrating this matching layer into both the transmitting and receiving transducers, the transmission pressure magnitude is increased by a factor of 8.7 and the overall system insertion loss is reduced by $30 \mathrm{~dB}$ [11].

\section{Payload Digital Circuitry}

The payload was capable of recording the full time domain signal from the the receiving transducer. A typical piezoelectric composite receiver signal is narrow band, with a centre frequency of $0.5-1 \mathrm{MHz}$. The payload used a dsPIC microcontroller from Microchip [14], which is a 16bit family of ICs with DSP functionality and relatively fast ADC's. The microcontrollers do not have a communications port fast enough to stream the ultrasonic signal in real time, so the signal must be buffered on-chip. The dsPIC33f706 [15] features a $1.1 \mathrm{MHz} 10 \mathrm{bit} \mathrm{ADC}$ and $16 \mathrm{kBytes}$ RAM. This can potentially store up to $7 \mathrm{~ms}$ of time domain data in RAM. The $A_{0}$ Lamb wave at $600 \mathrm{kHz}$ travels at approximately $3 \mathrm{~mm} / \mu \mathrm{s}$, so $7000 \mu$ s corresponds to $21 \mathrm{~m}$ of travel. There is a common misconception that the Nyquist sampling criterion states that the sampling frequency must be double the highest frequency component. This is only true if the signal contains frequencies right down to DC. If the signal has limited bandwidth, the sampling frequency only needs to be twice the bandwidth and not twice that of the highest frequency component [16]. It is therefore sufficient to sample the narrowband $600 \mathrm{kHz}$ at $1.1 \mathrm{MHz}$ and to reconstruct the signal in post processing.

\section{E. Transmitter Front End}

The host microcontroller generates a TTL square wave burst using an output pin controlled by a timer. This drives a BS108 MOSFET in drain-source configuration which switches a 100V DC voltage over the transducer. When the MOSFET is off, the supply voltage is present across the transducer. Activating the MOSFET shorts the transducer to ground. The high voltage is created using a specialised DC-DC convertor, the Q01-12 [17] that can create $100 \mathrm{~V}$ with a power rating of $1 \mathrm{~W}$.

\section{F. Payload Receiver Front-End}

The receiver front end used an AD8058 [18], configured as an inverting voltage amplifier to provide the initial gain stage. A common-source junction Field-Effect Transistor (FET) amplifier was used with an LC tuner to provide additional gain $(60 \mathrm{~dB})$ and to band pass filter the signal. The centre frequency of the filter being the resonant frequency of the LC oscillator. The amplifier gain was tuned so that receiver signals were matched to the ADC range to minimise the quantisation error. Clamping diodes ensured that large input signals did not damage the ADC circuitry.

The peak output from the receiving transducer was in the order of hundreds of nano volts for pitch-catch air-coupled Lamb wave inspection. Minimising interference with this very low amplitude signal was critical for performance.

\section{G. Testing the Complete System}

Figure 7 shows the signal captured from a local pitch-catch experiment using a $1 \mathrm{~mm}$ thick aluminium sample. The signal can confidently be identified as the $A_{0}$ Lamb wave mode due to its correct arrival time at $90 \mathrm{us}$. All systems were embedded on the robot and battery powered. The system did not required 
any external signal, allowing the robot to maintain its wireless capabilities. The overall SNR was approximately 23 after eight averages which were performed by the embedded microcontroller.
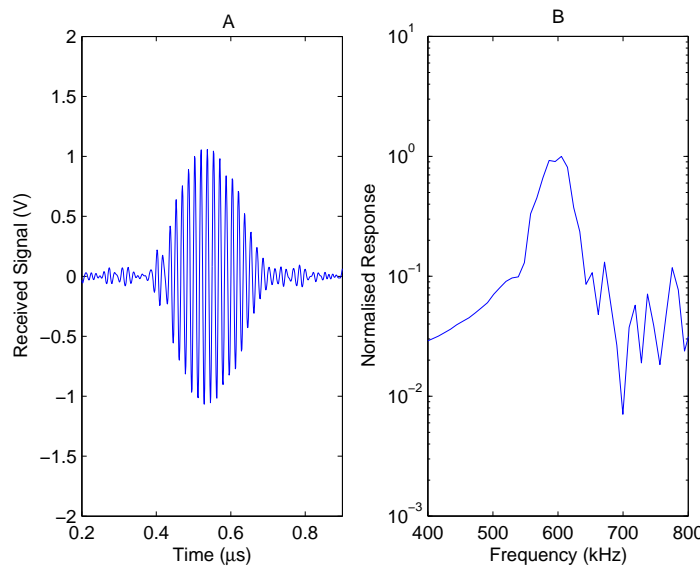

Fig. 7. Lamb wave transmitted and measured using the embedded payload showing time domain signals ' $\mathrm{A}$ ' and associated power spectrum 'B'

\section{H. Sensitivity to Surface Curvature}

The $A_{0}$ Lamb wave mode was preferentially excited when the excitation signal was matched, temporally and spatially, into the desired mode. As the radius of curvature of the sample decreases the matching condition is increasingly degraded leading to a lower amplitude guided wave in the sample. The experimental arrangement shown in Figure 8 was used to test the payload's sensitivity to curved surfaces. $1 \mathrm{~mm}$ aluminium samples were machined to have various different curvatures. The samples were fixed onto a flat surface and the width, w and height, $h$ of the arc were measured. The arc radius was calculated using Equation 1. The excitation was a 60V, 5 cycle tone burst at $600 \mathrm{kHz}$. The amplitude of the pitch-catch Lamb wave was recorded for propagation over $4 \mathrm{~cm}$, at the optimum incident angle.

$$
r=\frac{h}{2}+\frac{w^{2}}{8 h}
$$

The normalized receiver amplitudes are plotted against radius in Figure 9. As expected, the sensitivity to surface curvature is severe, with amplitude dropping off rapidly as the radius is reduced.

This result has two implications for the inspection of curved surfaces such as pipes.

- The reduction in Lamb wave amplitude will reduce the system's SNR.

- If the inspection is utilising signal amplitude as the measurement then the system will be cross sensitive to surface curvature. In addition to this, the reduction in Lamb wave amplitude is likely to be dependent on the robot's orientation relative to the surface.

Specimen curvature will have less effect on smaller transducers, since the coincidence principle only needs to hold

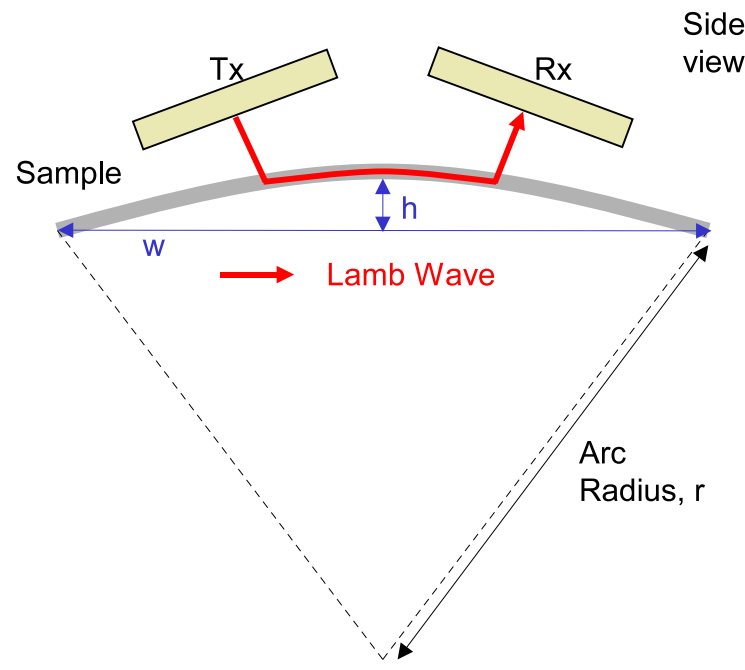

Fig. 8. Experiment to test the ultrasonic payloads sensitivity to curved surfaces. The transducers $30 \times 30 \mathrm{~mm}$ and were excited at $600 \mathrm{kHz}$. The sample was $1 \mathrm{~mm}$ thick aluminium.

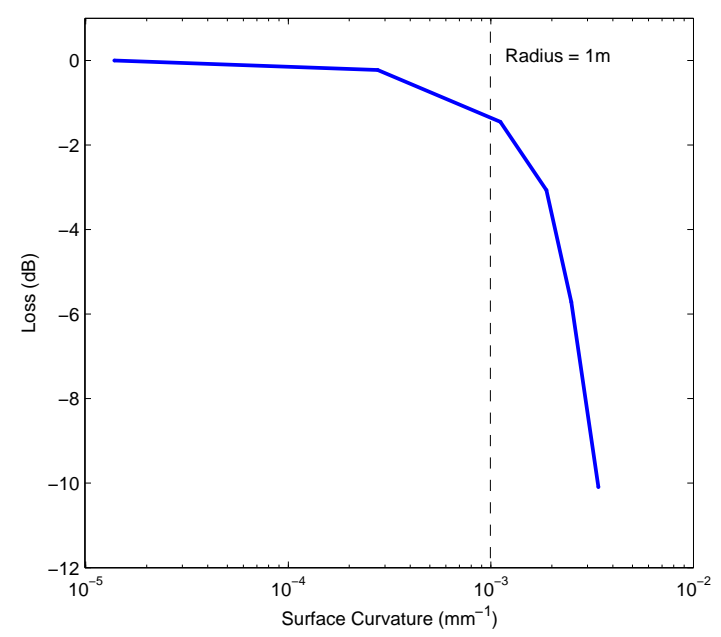

Fig. 9. Signal attenuation against the surface curvature. Surface curvature is defined as 1 divided by the radius shown in Figure 8

over a smaller area. Pipes are only curved along one axis, so the transducer shape can be optimised so that the dimension tangential to the pipe circumference is kept narrow and the dimension that follows the length of the pipe is extended. However, this would require careful alignment of the transducers relative to the pipe. Alternatively, the transducer could be split into an array of separately aligned elements, but this would dramatically increase the complexity of the system.

\section{Sensitivity to Material Thickness}

The angled transducers work most effectively with thin plate samples. As the plate thickness is increased, more energy is required to vibrate the plate and the magnitude of the vibration and hence receiver amplitude decreases. The ultrasonic payload was tested on a series of plate thicknesses ranging from $0.75 \mathrm{~mm}$ to $30 \mathrm{~mm}$. The transducers were positioned at the optimum angle for each sample, the excitation signal was a 
$60 \mathrm{~V}, 5$ cycle tone burst at $600 \mathrm{kHz}$. The receiver amplitude was plotted against sample thickness in Figure 10. Over the range $0.75 \mathrm{~mm}$ to $3 \mathrm{~mm}$, the signal amplitude decays in a fairly linear fashion. A $3 \mathrm{~mm}$ sample yields a $11 \mathrm{~dB}$ smaller receiver signal than a $0.75 \mathrm{~mm}$ plate. Above $5 \mathrm{~mm}$ the signal amplitude becomes almost constant as the $A_{0}$ and $S_{0}$ modes converge and the wave becomes more like a Rayleigh wave [3]. Rayleigh waves only propagate in the surface region of the material, so their amplitude is not dependent on sample thickness. It is interesting to note that the payload can propagate ultrasonic signals in a sample of any thickness, but that when the sample is thicker than $5 \mathrm{~mm}$, the wave only propagates in the surface region. In these thicker samples, the system will only be sensitive to defects in the upper region of the material. This can be compensated for by lowering the operating frequency, but depending on the experiment, may result in reduced resolution.

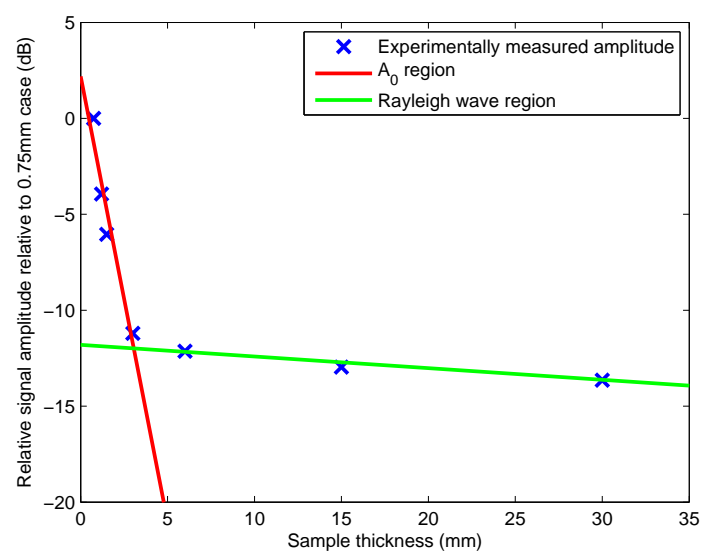

Fig. 10. Sensitivity to plate thickness (at optimum incidence angle). The solid lines are experimental best fit lines.

\section{Roвot Positioning}

In order to effectively interpret or combine a series of NDE measurements, the position where each measurement was taken is fundamental.

Consider a single robot instructed to perform a scan of a large plate. If the initial location is known, the RSA can estimate its movement by recording the motion of the two drive wheels, either by monitoring the motor drive signal or more accurately using wheel encoders. This is known as 'dead reckoning' and is the most basic form of localisation. The measurements are imperfect due to wheel slippage and other subtle noise sources [19]. These errors accumulate over time increasing the uncertainty in the position estimate. Although accurate over short periods, dead reckoning is not adequate for large scans. The problem of accumulating errors can be obviated by the use of absolute positional sensors such as a Global Positioning System (GPS). (Note that in this work GPS is used to represent any global positioning system and not necessarily satellite based GPS.) However, absolute positioning systems also contain uncertainty in their measurements and typically do not have the fidelity required for adequate tracking. In addition, absolute positioning sensors may only provide partial information, such as position, but not orientation.

It is possible to use a linear state-space approaches such as the Kalman filter [6] to combine the higher accuracy short term relative measurements with less accurate absolute measurements in such a fashion as to make optimal estimation of current position by combining the available evidence from the different sensors. The Kalman filter can be adapted to non-linear systems by linearizing about the current mean and covariance[20]. This approach is known as the extended Kalman filter and has been widely utilised in the robotics community [21], [22], [23], [24], [25].

An Extended Kalman filter was implemented on the miniature robotic vehicles using the Cricket Indoor Positioning System [5], [26] for global measurement and 300 count wheel encoders mounted on the robots two drive wheels for relative positional estimation. The Cricket system has a 1D measurement error of $1 \mathrm{~cm} / \mathrm{m}$. The maximum error in robot belief was approximately $10 \mathrm{~mm}$.

\section{Data Processing}

In the local pitch-catch arrangement, the two transducers were positioned side by side on a single robot, as shown in Figure 4 . They were angled to generate and receive the zeroth order antisymmetric Lamb wave mode $\left(A_{0}\right)$ in the specimen. The angle required for the appropriate mode conversion of the incident planar wave to $A_{0}$ can be calculated using Snell's law [12].

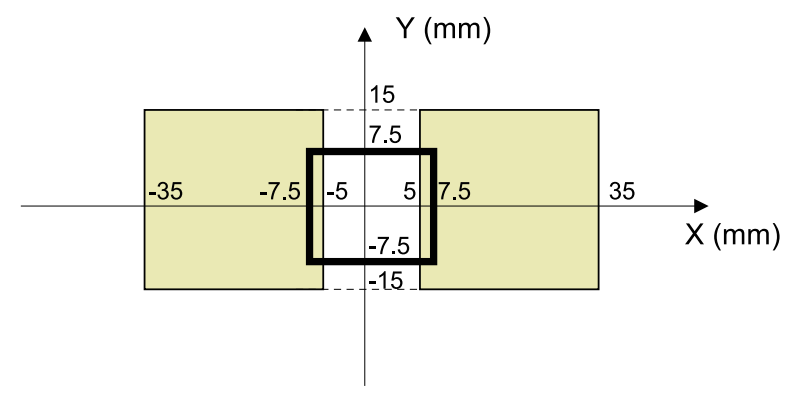

Fig. 11. Schematic diagram of local pitch-catch experiment showing specific transducer and artificial defect dimensions. The transducers were each $30 \times 30$ $\mathrm{mm}$ separated by $10 \mathrm{~mm}$. The angle relative to the $\mathrm{Z}$ axis (Figure 4 ) was only $9.8^{\circ}$, so the transducers each cover an area of approximately $30 \times 30 \mathrm{~mm}$. The defect was $15 \times 15 \mathrm{~mm}, 0.5 \mathrm{~mm}$ deep from the top surface. The sample was $1 \mathrm{~mm}$ thick aluminium.)

The $A_{0}$ Lamb wave mode was dispersive which meant that the phase velocity was dependent on the 'frequency thickness product'. A change in plate thickness and hence frequency thickness product, caused a change in phase velocity. Since the angle for optimum transition was dependent on phase velocity, a change in thickness meant that the angle was suboptimal and resulted in an attenuated signal. In order to generate the $A_{0}$ mode at $600 \mathrm{kHz}$ the angle was set at $9.8^{\circ}$ for $1 \mathrm{~mm}$ thick aluminium. The notable advantage of this sensing methodology was that the resolution is dependent on the transducer dimensions rather than the excitation signal, so good resolution is possible even at low frequencies. This arrangement is sensitive to defects at any point through the 
material thickness making it attractive over other techniques, such as visual inspection, that can only detect surface breaking defects. The main limitation with this approach is that in order to scan the entire surface with a fine resolution, the RSA must cover every point on the surface leading to very long scan paths.

\section{A. Sensors Sensitivity to Defects}

A high precision flatbed $2 \mathrm{D} \mathrm{X}-\mathrm{Y}$ scanner was used to decouple the effects of the finite transducers from the inherent positional uncertainty of the robots. In the following example the scanner was used to measure the payloads response to a $50 \%$ depth, $10 \times 30 \mathrm{~mm}$ defect in a $1 \mathrm{~mm}$ aluminium plate sample. The defect had rounded corners with a $5 \mathrm{~mm}$ radius on the XY plane. The payload was configured as shown in Figures 4 and 11 . The transducers were $600 \mathrm{kHz}, 30 \times 30$ $\mathrm{mm}$, held $10 \mathrm{~mm}$ apart at the appropriate incident angle to generate the $A_{0}$ Lamb wave mode. They were excited with a 10 cycle tone burst. The defect was positioned at $0,0 \mathrm{~mm}$ and an area of $120 \times 80 \mathrm{~mm}$ was scanned, this was divided into $5 \times 5 \mathrm{~mm}$ cells, and the payload was positioned at the corner of each cell. The time history for each receiver position was captured, enveloped using a Hilbert transform and the maximum amplitude extracted. The result is shown in Figure 12. The experimental results show a trough in receiver amplitude ranging from $\mathrm{X}=-50$ to $50 \mathrm{~mm}$ and $\mathrm{Y}$ ranging from -20 to $20 \mathrm{~mm}$. Since the defect is not large enough to completely obscure the transducer, it is probable that the difference in minimum amplitude is due to noise in the experimental results. This noise is so severe in the experimental results, that it actually hides the defect in some locations, such as $\mathrm{X}=-30 \mathrm{~mm}, \mathrm{Y}=10 \mathrm{~mm}$. The raw signal at this location is indistinguishable from the signal of a defect free region, suggesting that a Lamb wave has been transmitted.

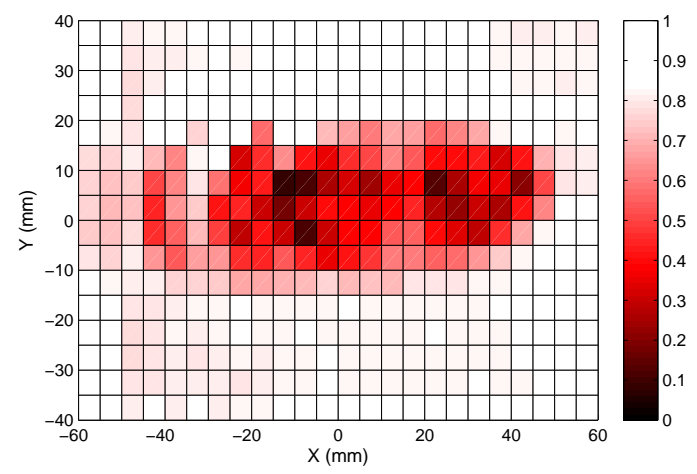

Fig. 12. This figure plots the experimentally measured normalized receiver amplitude at numerous points over the surface of a $1 \mathrm{~mm}$ aluminium sample with the artificial $10 \times 30 \times 0.5 \mathrm{~mm}$ defect in the center of the plate. The receiver amplitudes have been normalized relative to the defect free region of the plate.

\section{B. Local-Pitch Catch Imaging}

Previous work by some of the authors [27] integrated a probability of detection model into the Lamb wave scanner.
Several defect free plates were raster scanned. For each plate a probability density function was created that plotted the likelihood of receiving a change in received Lamb wave signal at any location on the plate. This was created by plotting the histogram of signal amplitude, irrespective of payload position. The results showed a Gaussian distribution centred around the mean received amplitude as illustrated in Figure 13. An amplitude threshold was then chosen at a $-3 \sigma$ deviation from the mean receiver amplitude for the defect free case (approximately 5\% of the maximum received amplitude in a $1 \mathrm{~mm}$ aluminium sample with eight averages in the time domain). Deviations beyond this threshold were taken to be indicative of a defect.

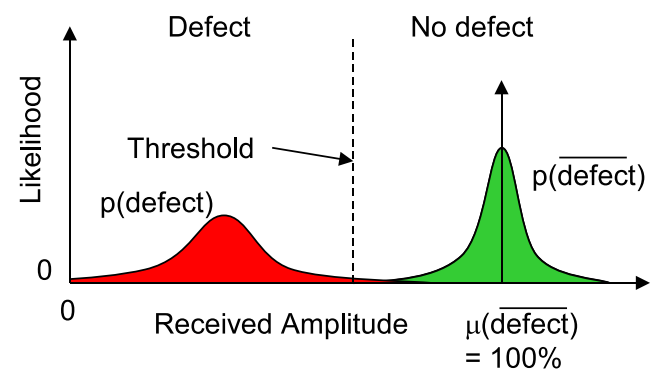

Fig. 13. The probability density function for air-coupled Lamb wave Payload over regular and defective regions

When this approach was integrated into the miniature robotic vehicle platform, inconsistent transducer alignment presented itself as a baseline shift in receiver amplitude. This was most prevalent if the sample was not perfectly flat, or the robot tyres perfectly uniform. (A $0.66^{\circ}$ missalignment in one direction causes a 50\% drop in received amplitude [28].) Since the missalignment can occur in both $X$ and $\mathrm{Y}$ axes, the tolerance is extremely tight. Unfortunately, the defect detection model was extremely sensitive to a receiver amplitude baseline shift. Consider Figure 14 where the upper diagram shows the ideal case; the defect is clearly identified by a dip in receiver amplitude. In the lower diagram there is an inconsistent amplitude baseline, caused by a slightly uneven plate sample. In this case it is impossible to set a satisfactory threshold. Threshold 1 is too high, incorrectly classifying most of the plate as defective. If the threshold is lowered to below the minimum baseline, the system becomes insensitive to all but the most extreme defects. This made the technique impractical to implement experimentally. An alternative approach is to map the amplitude of the transmitted Lamb wave. This obviates the need of a threshold and presents defects as sharp discontinuities in the image. This approach is implemented in the next section.

\section{The Implementation of Lamb Wave Scanning}

Farlow [7] demonstrated a Lamb wave scanner in his $\mathrm{PhD}$ thesis where he took the measurement location as the centre of the two transducers. This is the origin in Figure 4. In reality the measurement is spread over the transducer active area, which is $30 \times 70 \mathrm{~mm}$ for the payload described herein. The approach taken in this work was to divide the sample into a fine mesh 


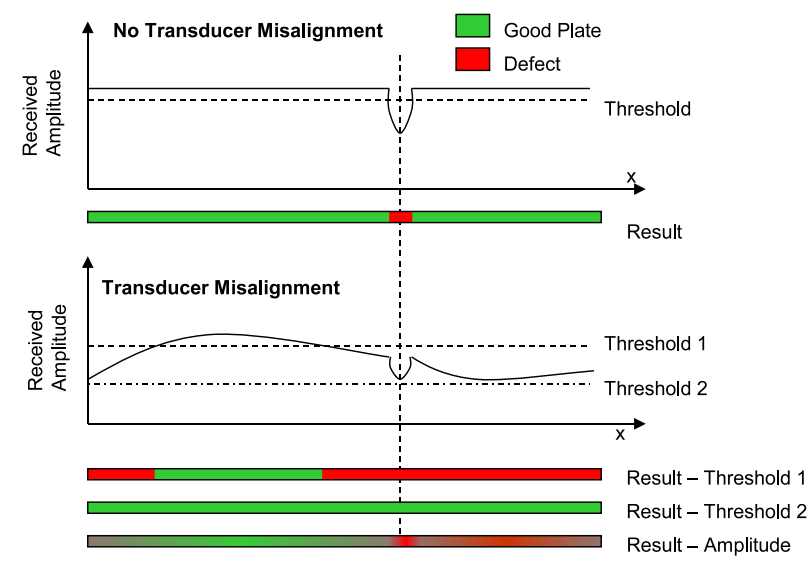

Fig. 14. The effect of misalignment of transducers on thresholding. The top diagram shows perfect alignment where thresholding works well. In the bottom diagram, small changes in the alignment result in poor defect detection

of cells and to apply new measurements to all relevant cells. This is shown in Figure 15 'A'. The approach obviates the need to sample in a regular grid, something that is straightforward when using a flatbed X-Y scanner, but more difficult when using the miniature robotic vehicles because of reduction in positional control. Mapping the receiver amplitude removes the need to set a threshold. Missalignment presents itself as a baseline shift that can be filtered out. The important limitation is that the sensor is unable to detect gradual changes in plate thickness, which are mistaken for poor alignment.

In this approach, the same cell is often measured multiple times. The effect is illustrated in Figure 15 ' $\mathrm{B}$ ' and ' $\mathrm{C}$ '. In ' $\mathrm{B}$ ', each row is sampled twice, once in the top half of the active area and once in the bottom. In this case, the sensible solution would be to take the mean of the two readings. The situation becomes more complicated in ' $\mathrm{C}$ ', where several cells are sampled numerous times.

Assuming the sensor alignment remains constant, the measured amplitude at the receiving end of the sensor is dependent on the efficiency of Lamb wave generation and reception which is disrupted if a defect is present under either of the transducers and to the extent of attenuation or scattering as the Lamb wave travels across the plate from the point of generation to the point of reception. With reference to Figure 4, consider the following extreme example. A through thickness crack orientated along the $\mathrm{Y}$ axis would completely block Lamb wave transmission and result in zero receiver amplitude. The same crack orientated along the $\mathrm{X}$ axis would have minimal effect on the Lamb wave and would result in a high receiver amplitude. That said, the region affected by the defect can be thought of as the spatial convolution between the defect and sensor footprints. The signal attenuation varies with the amount of the defect under the sensor. For defects that are smaller than the relatively large sensor head $(70 \times 30 \mathrm{~mm}$ in this case) the response typically leads to a trough shape in the received amplitude shown in Figure 16 'I'. The same effect is visible in Figure 12. The precise shape of the trough is dependent on the geometry of the defect, with smaller defects leading to sharper edges.
Instead of averaging measurements it was deemed preferable to weight them according to their position in the sensing area, favouring those in the centre where the response is likely to be greater. This is further supported by the physics of angled Lamb wave generation that produces a Lamb wave with high amplitude in the centre, tailing off toward the edges. The significant assumption made in this work is that, for ease of implementation, the trough shaped response can be approximated to a 2D Gaussian. The Gaussian can be represented using Equation 2:

$$
W(x, y)=\frac{1}{2 \pi \sigma_{\operatorname{sen}_{x}} \sigma_{\operatorname{sen}_{y}}} e^{-\frac{1}{2}\left(\frac{x^{2}}{\sigma_{\operatorname{sen}_{x}}{ }^{2}}+\frac{y^{2}}{\sigma_{\operatorname{sen}_{y}}{ }^{2}}\right)}
$$

where $W(x, y)$ is the weighting and $x$ and $y$ are defined relative to the sensor's origin (Figure 4 ), $\sigma_{s e n_{x}}$ and $\sigma_{\text {sen }_{y}}$ were used to shape the Gaussian and were chosen to approximate the $2 \mathrm{D}$ shape in Figure 12. These can be tuned if a specific size of defect was expected.

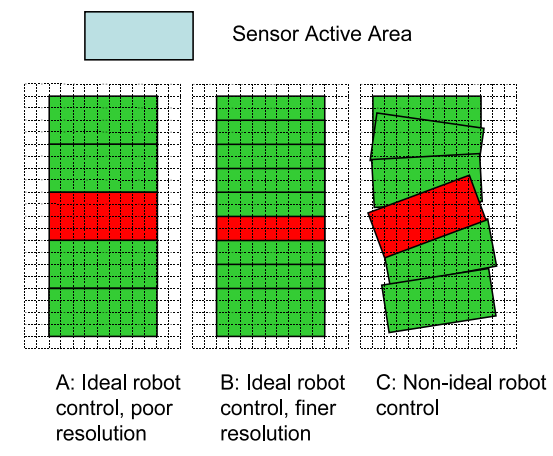

Fig. 15. Examples of overlapping measurement regions

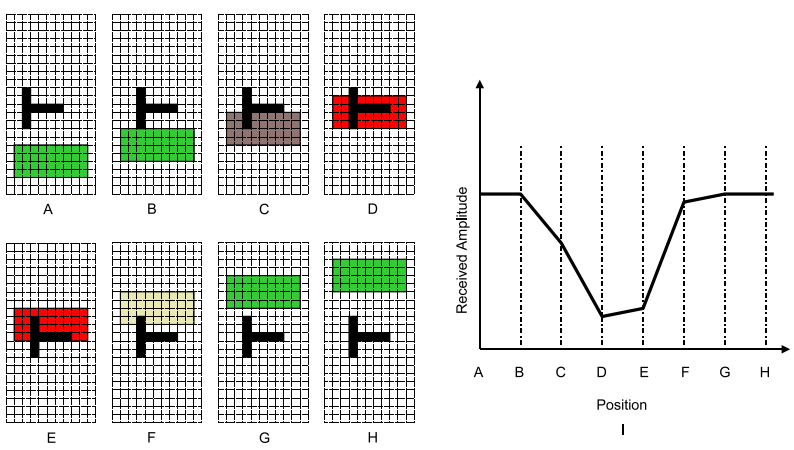

Fig. 16. Graphical representation of a Lamb wave pitch-catch sensor passing over an artificial defect.

Each cell in the sample mesh retains a history of each amplitude/weighting pair for every measurement that covers the cell. The final cell value is calculated as a weighted average of the amplitude data as shown:

$$
A(x, y)=\sum_{n=0}^{N}\left[\frac{W_{n}(x, y)}{\sum_{m=0}^{M} W_{m}(x, y)} A_{n}(x, y)\right]
$$

As discussed in Section IV the robot's position contains a degree of uncertainty. This uncertainty is captured by the Kalman filter covariance matrix [25]. Assuming the states X, Y 
and $\theta$ are mutually independent, i.e. all terms in the covariance are negligible except for the diagonal containing the variances, it is straightforward to refine the sensor weighting to account for the robot positional uncertainty. The robot's positional uncertainty is defined by three variances $\sigma_{\text {robot }_{x}}^{2}, \sigma_{\text {robot }_{y}}^{2}$ and $\sigma_{\text {robot }_{\theta}}^{2}$ defining uncertainty in $\mathrm{X}$ and $\mathrm{Y}$ axis and orientation respectively.

The positional uncertainty was integrated in two stages. The first stage of which was to convolve the positional uncertainty for $\mathrm{X}$ and $\mathrm{Y}$ with the sensor model, which can conveniently be expressed as another Gaussian function:

$$
W(x, y)=\frac{1}{2 \pi \sigma_{x} \sigma_{y}} e^{-\frac{1}{2}\left(\frac{x^{2}}{\sigma_{x}^{2}}+\frac{y^{2}}{\sigma_{y}^{2}}\right)}
$$

where:

$$
\sigma_{x}^{2}={\sigma_{\text {sen }}}^{2}+{\sigma_{\text {robot }}}^{2}, \quad \sigma_{y}{ }^{2}={\sigma_{\text {sen }}}^{2}+\sigma_{\text {robot }_{y}}{ }^{2}
$$

The second stage was to integrate the uncertainty in the robot heading, $\theta$. Since the sensor module is mounted in front of the robot, the transform from the robot coordinate system to the sensor coordinate system is simply a $+75 \mathrm{~mm}$ translation along the $\mathrm{Y}$ axis. When the robot turns on the spot, the sensor payload follows a circular path of $75 \mathrm{~mm}$ radius.
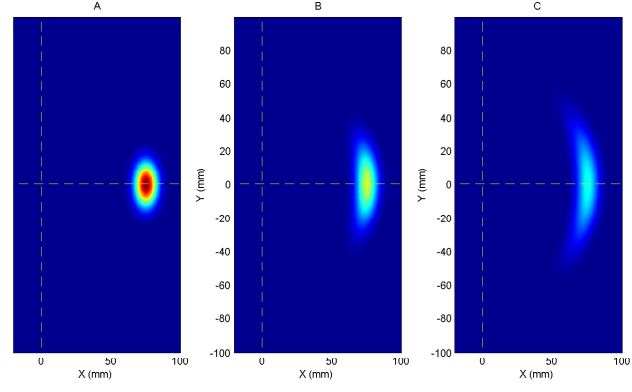

Fig. 17. The skewing of the Gaussian probability density function of a defective region when angular uncertainty is considered. In 'A' there is no angular uncertainty, in ' $\mathrm{B}$ ' the angular uncertainty has a variance of $2^{\circ}$ and in ' $\mathrm{C}$ ' the angular uncertainty has a variance of $5^{\circ}$.
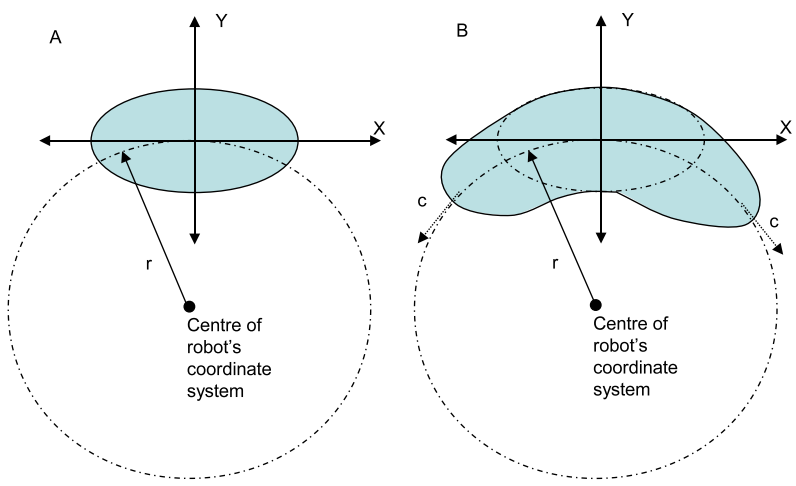

Fig. 18. The effect of angular uncertainty on the measurement probability density function. In A there is no angular uncertainty. The small angular uncertainty in B, skews the Gaussian function.

Figure 17 shows the effect of angular uncertainty on cell weightings. The total sum of the individual weights remains constant, but as angular uncertainty increases the weighting is spread further along the arc. This effect can be thought of as the summation of a series of the 2D Gaussian functions calculated by Equation 4 at a range of angles, weighted by the angular uncertainty, $\sigma_{\text {robot }_{\theta}}$. This is shown graphically in Figure 18. It can be represented mathematically as follows:

$$
W(x, y)=\sum_{\theta}=-\pi^{\pi} \frac{1}{\sigma_{\text {robot }_{\theta}} \sqrt{2 \pi}} e^{\frac{-1}{2} \frac{\theta^{2}}{\sigma_{\text {robot }_{\theta}}^{2}}} P\left(x^{\prime}, y^{\prime}\right)
$$

Where:

$$
\left[\begin{array}{l}
x^{\prime} \\
y^{\prime}
\end{array}\right]=\left[\begin{array}{cc}
\cos (\theta) & -\sin (\theta) \\
\sin (\theta) & \cos (\theta)
\end{array}\right]\left[\begin{array}{l}
x \\
y
\end{array}\right]+\left[\begin{array}{c}
0 \\
y_{0}
\end{array}\right]
$$

and $W\left(x^{\prime}, y^{\prime}\right)$ is calculated from Equation 4 , using $x^{\prime}$ and $y^{\prime}$ from Equation 6.

It should also be noted that the maximum measured Lamb wave amplitude (observed in undamaged plate specimens) is a function of the specimen's material and thickness. The algorithm presented herein works with received amplitudes normalised to this maximum, so is reasonably generic to samples of different material or thickness. However as discussed in Section III-I, increasing the material thickness causes the ultrasonic wave to tend towards a Rayleigh wave at which point the system would only be sensitive to defects near the top surface of the sample. In addition to this, increasing the thickness of the sample reduces the amplitude of material displacement, which in turn reduces the system's SNR.

As discussed in Section III-H, the sensor is cross sensitive to sample curvature. However, if the curvature is constant on one axis, such as a pipe, our approach is effective as long as sensor is constantly aligned to the curved surface throughout the inspection. Inspecting curved surfaces will result in a constant reduction of the sensor output and will distort the shape of the sensor model, this is best illustrated through an example. Consider a straight pipe of uniform diameter and wall thickness, taking the axes defined in defined in Figure 11. The sensor can be orientated so that its $\mathrm{X}$ axis is aligned with an axis running along the centre of the pipe. A constant reduction in the measured maximum Lamb wave amplitude relative to a flat plate will be observed due to suboptimal excitation and measurement, but this has little effect (other than reducing SNR) as the algorithm processes normalised amplitudes. The sensor model, as approximated by a 2D Gaussian function, will remain unchanged in the $\mathrm{X}$ axis, but needs to be 'squeezed' in the $\mathrm{Y}$ axis (relative to the sensor model for a flat sample) to reflect decreasing quality of Lamb wave generation and reception as the distance from the $\mathrm{X}$ axis increases (due to increasing misalignment of the sensor as a result of surface curvature.) This can be accounted for by adjusting the parameters that define the shape of 2D Gaussian approximation. The approach is currently poorly suited to complex sample geometries, unless the curvature is small enough that effect can be considered a source of noise. 


\section{Integration into the Hardware Platform}

Each cell in the sample grid retains a history of each measurement/weighting pair. The spatial 'weighting' distribution is calculated for each ultrasonic measurement using the Kalman Filter's estimate of positional uncertainty at the last filter update. The resulting cell attenuations are calculated as a weighted average of all measurements using Equation 3. In practise, measurements with negligibly low weighting are discarded to save memory (the weighting is stored as 8 bits in memory, so weightings below 1/255 are discarded).

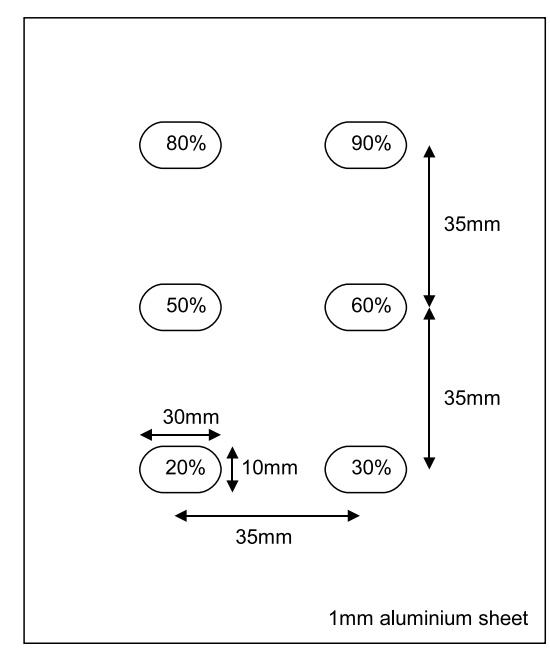

Fig. 19. Schematic of sample for local-pitch catch experimental scan, containing $630 \times 10 \mathrm{~mm}$ defects. The defect depth is shown as a percentage of material depth.

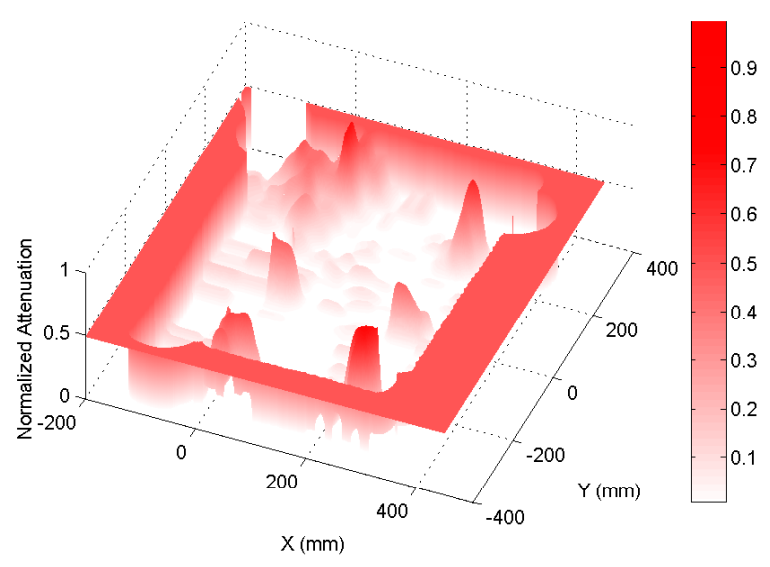

Fig. 20. Example scan using of $1 \mathrm{~mm}$ using air-coupled pitch-catch transducers shown in $3 \mathrm{D}$. The $\mathrm{Z}$ axis plots the ultrasonic signals attenuation at each point.

The robot described in section II was equipped with the payload described in section III. The weighted average algorithm was integrated into the robots Graphical User Interface. The robot was instructed to scan a $1 \mathrm{~mm}$ aluminium plate with six artificial defects, each $10 \times 30 \mathrm{~mm}$ in size and of varying depth ranging from 20 to $90 \%$. The geometry of the sample is shown in Figure 19 with Figure 20 showing the resulting image of normalized reception attenuation. A peak in amplitude toward 1 represents large signal attenuation indicative of a defect. Unscanned regions are assigned an amplitude of 0.5 , regions that have been scanned and shown as defect free, have an amplitude approaching 0 (no attenuation). All six defects were clearly visible.

\section{CONCLUSION}

A non-contact ultrasonic inspection system has been presented. The system comprises of one or more miniature robotic vehicles each carrying a non-contact ultrasonic inspection payload capable of generating and measuring the $A_{0}$ Lamb wave mode in thin plate specimens. This work illustrates the effects of the large sensing aperture and robot positional uncertainty on the fidelity of the resulting scan and presented an algorithm to combine a series of spatially sampled measurements, each with a degree of uncertainty in both the measurement and the position in an optimum fashion. A sensor model is required to relate the ultrasonic attenuation measurement to the probability of a defect being present. However, the sensitivity of sensor output to defect geometry makes this impractical if there is no a priori knowledge of the defect. This sensitivity to defect geometry means that the system is not capable of measuring precise information about the defect, such as the depth, and is therefore only suitable as a screening method. Areas of concern need to be examined by other means to access the structural integrity of the plate. The relatively large sensor head $(70 \times 30 \mathrm{~mm})$ limits the finest possible resolution of the sensor to $70 \mathrm{~mm}$ in one axis and $30 \mathrm{~mm}$ in the complementary axis if the sensor is aligned the axis system. This is the minimal distance in which two discrete defects can be distinguished. The system is capable of detecting defects with much smaller geometries (such as $5 \mathrm{~mm}$ radius, $0.5 \mathrm{~mm}$ deep), but cannot separate two such defects located in close proximity. A significant improvement in resolution will come from a reduction in the size of the transducers. However, a reduction in transducer size reduces the active area, making it extremely difficult to achieve acceptable signal to noise ratio.

The robot positional uncertainty of up to $10 \mathrm{~mm}$ is large relative to the size of the area scanned. This results in a further reduction in resolution as the image becomes blurred as the algorithm applies measurements to all possible robot locations. The high positional uncertainty is a result of the relatively low accuracy of the Cricket [26] global positioning system used to track the robots. Although alternative systems such as VICON [29] can dramatically reduce this error in a laboratory environment, there is no universal solution to robot positioning in realistic environments and positional uncertainty will be a factor in most applications. A key accomplishment of this work is the ability to produce useful results even with significant positional uncertainty.

Finally, future work could investigate the use of guided waves to characterise the specimen's elastic properties in an effort to extract additional information such as structural loading. It will also consider the use of long range guided wave inspection to perform pulse-echo measurements, speeding up the inspection and rapidly reducing the distance that must be travelled by the robot. 


\section{ACKNOWLEDGMENT}

This research received funding from the Engineering and Physical Sciences Research Council (EPSRC) and forms part of the core research program within the Research Centre for Non-Destructive Testing (RCNDE), in the UK.

\section{REFERENCES}

[1] JM Farley and M. Babcock. Best Practice in the Application of NDT An Update. In World Conference on NDT, 2004.

[2] R. Singh. Three decades of NDI reliability assessment. Contract, 41608(99-C):404, 2000.

[3] I. A. Viktorov. Rayleigh and Lamb waves: Physical theory and applications. Plenum Press, 1967.

[4] R. Farlow and G. Hayward. An automated ultrasonic NDT scanner employing advanced air-coupled 1-3 connectivity composite transducers. Insight, 38(1):41-50, 1996.

[5] A. Smith, H. Balakrishnan, M. Goraczko, and N. Priyantha. Tracking moving devices with the cricket location system. Proceedings of the 2nd international conference on Mobile systems, applications, and services, pages 190-202, 2004.

[6] R.E. Kalman. A new approach to linear filtering and prediction problems. Journal of Basic Engineering, 82(1):35-45, 1960.

[7] R. Farlow and G. Hayward. Real-time ultrasonic techniques suitable for implementing non-contact NDT systems employing piezoceramic composite transducers. Insight, 36(12):926-935, 1994.

[8] A. Gachagan, G. Hayward, SP Kelly, and W. Galbraith. Characterization of air-coupled transducers. Ultrasonics, Ferroelectrics and Frequency Control, IEEE Transactions on, 43(4):678-689, 1996.

[9] CB Scruby and LE Drain. Laser ultrasonics: techniques and applications. Taylor \& Francis, 1990.

[10] S. Dixon and SB Palmer. Wideband low frequency generation and detection of Lamb and Rayleigh waves using electromagnetic acoustic transducers (EMATs). Ultrasonics, 42(10):1129-1136, 2004.

[11] Gordon Hayward Stephen P. Kelly and Tomas E. Gomez AlvarezArenas. Characterization and assessment of an integrated matching layer for air-coupled ultrasonic applications. IEEE transactions on ultrasonics, ferroelectrics, and frequency control, 51:no. 10, 2004.

[12] J.L. Rose. Ultrasonic Waves in Solid Media. Cambridge University Press, 1999.

[13] Stephen P. Kelly. An Air Coupled Ultrasonic Array Scannng System for Rapid Through Transmission NDT. PhD thesis, University of Strathclyde, 2000.

[14] Microchip. http://www.microchip.com/, Accessed Sept 2009.

[15] Microchip. dsPIC DSC High-Performance 16-Bit Digital Signal Controllers, rev 1 edition, 2005.

[16] J.G. Proakis, D.G. Manolakis, DG Manolakis, and JG Proakis. Digital signal processing: principles, algorithms, and applications, volume 3 . Prentice Hall New Jersey, 1996.

[17] EMCO. Ultra-Miniature DC to HV DC Converters.

[18] Analog Devices. AD8058: Low Cost, Dual, High Performance Voltage Feedback, $325 \mathrm{MHz}$ Amplifier, rev b edition, August 2003.

[19] J. Borenstein and L. Feng. Measurement and correction of systematic odometry errors in mobilerobots. Robotics and Automation, IEEE Transactions on, 12(6):869-880, 1996.

[20] G. Welch and G. Bishop. An Introduction to the Kalman Filter. University of North Carolina at Chapel Hill, Chapel Hill, NC, 1995.

[21] S. Thrun, D. Fox, W. Burgard, and F. Dellaert. Robust Monte Carlo localization for mobile robots. Artificial Intelligence, 128(1-2):99-141, 2001.

[22] R. Lastra, P. Vallejos, and J. Ruiz-del Solar. Self-localization and ball tracking for the robocup 4-legged league. In Proceeding of the 2nd IEEE Latin American Robotics Symposium LARS, 2005.

[23] S. Shoval, I. Zeitoun, and E. Lenz. Implementation of a Kalman filter in positioning for autonomous vehicles, and its sensitivity to the process parameters. The International Journal of Advanced Manufacturing Technology, 13(10):738-746, 1997.

[24] SI Roumeliotis and GA Bekey. Collective localization: a distributed Kalman filter approach tolocalization of groups of mobile robots. In IEEE International Conference on Robotics and Automation, 2000. Proceedings. ICRA'00, volume 3, 2000.

[25] S. Thrun, W. Burgard, and D. Fox. Probabilistic Robotics. MIT Press, 2005.

[26] N.B. Priyantha. The Cricket Indoor Location System. PhD thesis, Massachusetts Institute of Technology, 2005.
[27] R. Summan, G. Dobie, J. Hensman, S.G. Pierce, and K. Worden. A probabilistic approach to robotic nde inspection. In Proceedings of 2009 Review of Progress in Quantitative Nondestructive Evaluation, Chicago, 2009.

[28] M. Castaings and P. Cawley. The generation, propagation, and detection of Lamb waves in plates using air-coupled ultrasonic transducers. The Journal of the Acoustical Society of America, 100:3070, 1996.

[29] Vicon . http://www.vicon.com, Accessed Sept 2009. 